\title{
The Life Sciences Mass Spectrometry Research Unit
}

\author{
Gérard Hopfgartner ${ }^{\star}$ and Emmanuel Varesio
}

\begin{abstract}
The Life Sciences Mass Spectrometry (LSMS) research unit focuses on the development of novel analytical workflows based on innovative mass spectrometric and software tools for the analysis of low molecular weight compounds, peptides and proteins in complex biological matrices. The present article summarizes some of the recent work of the unit: i) the application of matrix-assisted laser desorption/ionization (MALDI) for mass spectrometry imaging (MSI) of drug of abuse in hair, ii) the use of high resolution mass spectrometry for simultaneous qualitative/quantitative analysis in drug metabolism and metabolomics, and iii) the absolute quantitation of proteins by mass spectrometry using the selected reaction monitoring mode.
\end{abstract}

Keywords: Mass spectrometry

\section{Introduction}

Analytical chemistry has integrated itself in many fields of life sciences for the qualitative and quantitative analysis of molecules and elements. In pharmaceutical sciences, it is essential to follow the fate and the biotransformation of drugs in biological systems, as well as the monitoring of pharmacological and toxicological effects at a molecular level. The tremendous new developments in ionization techniques and mass analyzers over the last decade in particular the high sensitivity, the high-throughput capabilities and the hyphenation to separation sciences have brought mass spectrometry to the forefront of analytical techniques. Nowadays, analytical sciences and mass spectrometry are more and more considered as commodities in many fields such as chemistry, biology, medicine or pharmaceutical sciences. Despite the fact that high quality and robust analytical tools are commercially available, their operation has become more complex, requiring more training to fully exploit their features and optimize their running cost. The need for high quality quantitative and qualitative information is

\footnotetext{
${ }^{*}$ Correspondence: Prof. G. Hopfgartner School of Pharmaceutical Sciences University of Geneva, University of Lausanne Life Sciences Mass Spectrometry

Quai Ernest-Ansermet, 30

$\mathrm{CH}-1211$ Geneva 4

Tel.: +4122379 6344

Fax: +4122379 3332

E-mail: Gerard.Hopfgartner@unige.ch
}

particularly key in the fields of -omics and is challenging analytical sciences in terms of instrumentation, data acquisition workflows and data processing.

The endeavor of the Life Sciences Mass Spectrometry (LSMS) research unit is the development of novel analytical workflows based on innovative mass spectrometric and software tools for the analysis of low molecular weight compounds, peptides and proteins in complex biological matrices. Long-term expertise is available for non-regulated and regulated bioanalysis. Concepts for integrated sample collection (blood, plasma, urine, saliva) and analysis based on dried blood spot (DBS) for offline or on-line analysis have been recently developed and are currently evaluated.

The present article summarizes some of the recently published work of the group in the fields of i) mass spectrometry imaging, ii) metabolism, metabolomics and QUAL/QUAN approaches and iii) quantitative protein analysis by selected reaction monitoring MS.

\section{Recent Work}

\subsection{Mass Spectrometry Imaging}

Atmospheric pressure ionization mass spectrometry (MS) combined with liquid chromatography (LC) has mostly supplemented MS direct surface analysis techniques such as matrix-assisted laser desorption ionization (MALDI) due to the additional separation dimension. For LC-MS analysis most samples need to be homogenized, extracted and reconstituted in a solution and therefore the molecular spatial distribution information will be lost. However, for the analysis of low molecular weight compounds (e.g. pharmaceuticals, lipids, etc.), peptides and pro- teins in tissues samples, molecular spatial information remains of prime importance to understand biological processes.

Mass Spectrometry Imaging (MSI) is a label-free technique and relies on desorption/extraction followed by ionization of molecules present on the surface of a solid flat sample. By recording the massto-charge ratio $(\mathrm{m} / \mathrm{z})$ signal of molecules of interest at each spatial location (i.e. pixel), a 2D map is reconstituted as the image of their distribution within the sample. ${ }^{[1,2]}$ MALDI and SIMS have played a major role in the development of MSI on various MS platforms including ion trap, time-offlight (TOF) and Fourier Transform Ion Cyclotron Resonance (FT-ICR) while ambient ionization techniques (e.g. DESI, LAESI, LAPI) are growing of importance.

In our laboratory, MALDI with a highrepetition laser combined to a triple quadrupole linear ion trap $\left(\mathrm{QqQ}_{\mathrm{LIT}}\right)$ mass spectrometer has been described as a powerful analytical platform for the quantitative and qualitative analysis of low molecular weight compounds (LMWC) in biological fluids and tissue. ${ }^{[3-5]}$ This MS platform is particularly attractive for tissue imaging due to its ability to perform high sensitive and high-speed target analysis in the selected reaction monitoring (SRM) followed by confirmatory analysis using various trap MS/MS and $\mathrm{MS}^{3}$ acquisition modes. ${ }^{[3-5]}$ While brain, kidney and liver are the most investigated tissue to monitor drugs, peptides, or proteins distribution, MSI can be used with any type of sample. The analysis of drugs and their metabolites in body fluids can reveal recent drug of abuse intake, but only hair analysis can reveal chronological information about habitual consumption. Current hair analysis is mainly based on gas or liquid chromatography coupled to MS(/MS), but requires multiple 


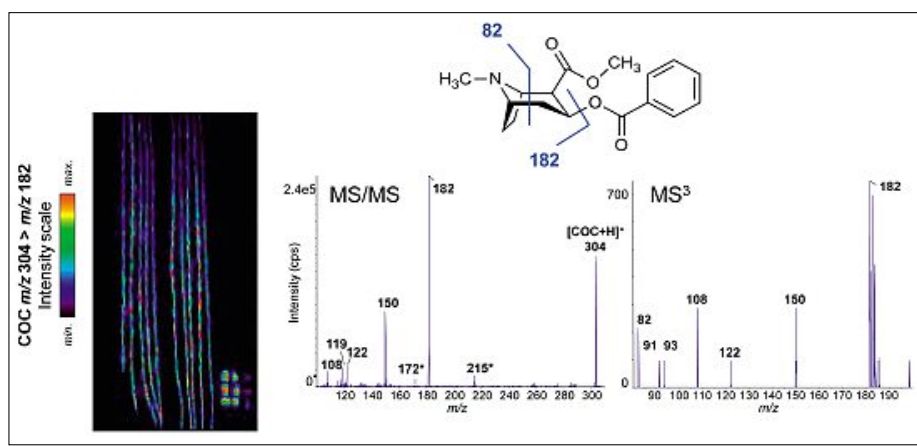

Fig. 1. Cocaine MALDI-SRM distribution in single hair with confirmatory MS/MS analysis. From ref. [6] with permission. Copyright American Chemical Society 2011. sample preparation steps beforehand (e.g. washing, pulverization, overnight hair digestion, extraction, derivatization). To generate time-resolved information about drug intake, hair samples have to be cut typically into several $1 \mathrm{~cm}$-long segments, which are pulverized and analyzed to reconstitute the drug consumption from scalp to hair tip (i.e. from present to past). This procedure is tedious and prone to analytical errors. MALDI-MSI was applied successfully to monitor drug of abuse in a single hair. The sample preparation procedure consisted in placing the hair on MALDI target plate and spraying $\alpha$-cyano-4-hydroxycinnamic acid matrix on the sample. As shown in Fig. 1, cocaine and its metabolites benzoylecgonine, ethylcocaine and norcocaine could be monitored on a single hair with a resolution of $1 \mathrm{~mm} .{ }^{[6]}$ The time analysis of an intact single hair sample of $6 \mathrm{~cm}$ (corresponding to several months of growth) was approximately of $6 \mathrm{~min}$. Resolution down to $0.1 \mathrm{~mm}$ can be used but on cost of time. MALDI-MSI can provide relative quantitative information when using adequate internal standard. Absolute accurate quantitation in hair remains challenging because of the lack of suitable calibration sample. Many strategies are being currently investigated to optimize absolute quantitation. While MSI is becoming an essential tool in life sciences for molecular imaging (cancer research, forensic, pathology, drug discovery) it is still in its infancy and many analytical aspects need to be further investigated and standardized. New developments in ambient ionization techniques such as DESI or liquid extraction surface analysis (LESA) in combination with ion mobility mass spectrometry[7] are still expanding the application of MSI.

\subsection{Metabolism, Metabolomics and QUAL/QUAN Approaches}

To better understand the pharmacology and toxicology of drugs, there is an intrinsic interest to analyze simultaneously the time/concentration profile of drugs and their metabolites, as well as the changes in endogenous metabolites. This task is particularly challenging because it requires accurate and precise quantitative analysis of several analytes and unambiguous iden- tification of biomarkers and metabolites only based on mass spectrometry. While triple quadrupole mass analyzers are mostly used to support quantitative analysis, high-resolution mass spectrometry is gaining of importance for drug metabolism and metabolomics investigations. In most cases these investigations are performed on the same set of samples but with different approaches.

The monitoring of endogenous metabolites and the identification of specific biomarkers can provide a better molecular understanding of a disease state, drug efficacy or toxicity and therefore is of great interest in drug discovery and development. In addition to other analytical techniques, liquid chromatography combined with mass spectrometry offers unique features regarding quantitation and identification of biomarkers candidates. The large vari-

\section{Metabolomics LC-MS(/MS) platform}

1. Sample handling

Sample collection, storage and stability

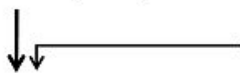

2. Analytical treatment Sample preparation, RP/HILIC chromatography and TOF-MS analysis<smiles>C[14CH3]</smiles>

3. Quality assessment \& Multivariate analysis

Repeatability, t-test, Principal Component Analysis (PCA)

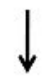

4. Data reduction by PCVG \& Groups interpretation Principal Component Variable Grouping(PCVG),

Removal of isotopes, adducts, etc. by careful interpretation of the groups from PCVG

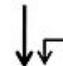

MZS Sarcher searches simultaneously through different DB (e.g. KEGG, HMDB, home-made, etc)

\section{List of candidates (biomarkers)}

Final list of $\langle\mathrm{RT}, m / z\rangle$ pairs with a reduced number of proposed compounds (Mw, structure, etc.)<smiles>C[14CH3]</smiles>

\section{Biomarker identification}

Fraction collection \& MS/MS experiments for structural confirmation (MS/MS spectra database search or manual spectrum interpretation) ety and concentration distribution of metabolites require the use of several analytical methods and can generate datasets containing a high number of variables for a relatively few number of samples analyzed. Atmospheric pressure ionization techniques in positive and negative mode, as well as the selection of complementary retention mechanisms in HPLC, enable the separation of broader range of compounds ding to their polarity. In the analytical sequence, samples have to be randomly analyzed to avoid any unrelated effect comfrom systematic instrument changes. In addition, measures should be taken by the analyst to control sample preparation efciency or analytical performance deterioration (e.g. column and MS interface clogging). For the comparison of large sets of from various samples acquired from different time points, multivariate analysis (MVA) plays a key role. In the case of LCS, metabolomics data processing generates a large amount of features consisting of $<$ retention time, $m / z>$ pairs. Application of MVA at that stage would generate poor quality data. Pre-processing steps are required to reduce chemical noise and to correctly detect and align the features over all samples. All these detected features correspond to potential candidate ions, isotopes, adducts, fragments, etc. which make interpretation harder as they have to be correlated for efficient identification. While the 
LC-MS is quite straightforward, detailed interpretation of the results (i.e. <retention time, $m / z>$ pairs) is much more timeconsuming and needs to take into account mass spectrometry specifics. In the LSMS research unit, a major analytical and informatics effort is undergoing to integrate mass spectrometry specifics in metabolomic data processing workflows as depicted in Fig. 2. ${ }^{[8]}$ Contrary to supervised correlation tools, principal component variable grouping (PCVG) was described as an unsupervised technique, which finds variables that have similar profiles (expression patterns) across all samples. These patterns can not only reveal variables that cause expected differences, but can also show unexpected behavior and artifacts that may confound or invalidate the experiment. This approach assigns the variables to a much smaller number of related groups that are often easier to understand, although the detailed spectral analysis is still required to determine the real molecular ions detected. Finally, structural confirmation of these biomarkers is achieved by MS and MS/MS experiments after LC fraction collection using ultra-high resolution and ion mobility mass spectrometry to provide high-quality MS/MS spectra suitable for library search or de novo spectra interpretation.

Because of the limited sample volume and the need of high-throughput capabilities, alternative approaches including targeted and not targeted LC-MS approaches based on high-resolution mass spectrometry with resolving power above 20,000 in combination with fast chromatography for QUAL/QUAN analysis are currently being investigated. ${ }^{[9]}$ Particularly attractive for QUAL/QUAN investigations, a novel independent acquisition mode referred as Sequential Window acquisition of All THeoretical fragment ion spectra (SWATH) or Global Precursor ions Scan mode (GPS) is currently developed for LMWC analysis (i.e. metabolism, metabolomics studies). Using a collision energy ramp, sequential precursor ions windows (typically $20 \mathrm{~m} / \mathrm{z}$ ) are used to collect in the same spectrum precursor and fragment ions. Several key information can be extracted from a single spectrum such as elemental formulae and fragment ions for qualitative structural assignments, while precursor ions or fragments can be used for quantitation using high resolution selected ion or reaction processing. SWATH or GPS were found to be superior to information dependent acquisition or $\mathrm{MS}^{\mathrm{ALL}}$ in combination with UHPLC (with analyte peak width of a few seconds) for qualitative analysis, but they require a fast acquiring mass spectrometer such as a Quadrupole-Time-of-Flight $(<50$ $\mathrm{msec}$ ). The analysis of talinolol and its hydroxylated metabolites using the SWATH

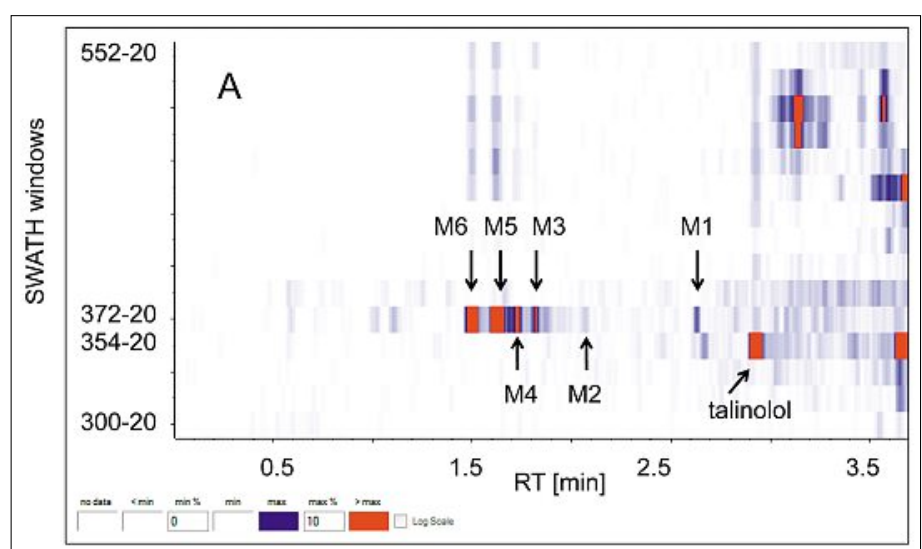

Fig. 3. A) Heat Map of SWATH experiments (15 product ion experiments) of the LCMS analysis after two hours incubation of talinolol in rat liver microsomes. B) SWATH spectrum of the peak eluting at $1.7 \mathrm{~min}$ corresponding to the M4 hydroxylated metabolite of talinolol. Adapted from ref. [9] with permission. Copyright Springer 2011.

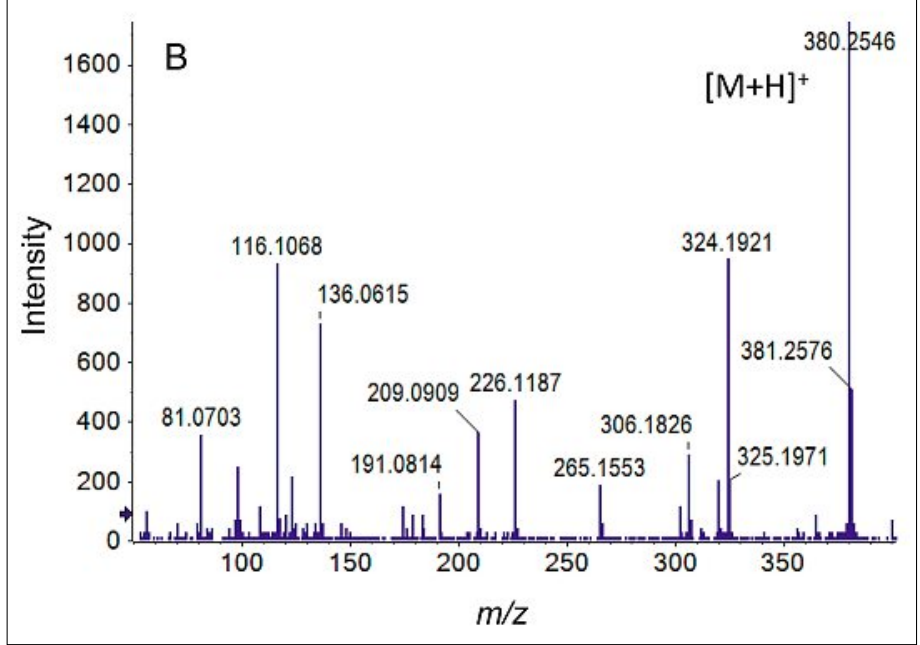

mode is illustrated in Fig. 3A. Because 15 MS experiments are recorded (in less than $400 \mathrm{msec}$ ) one of the convenient way to display the data is a heat map. A typical SWATH MS spectrum is depicted in Fig. 3B.

The success of LC-MS relies mostly in the orthogonality of both techniques. In complex samples from biological systems, the chromatographic peak capacity is often insufficient or the mass spectrometer cannot differentiate between isobaric metabolites. To improve the peak capacity, comprehensive 2D-LC separation can be considered but this approach requires a very fast second dimension. For decades, ion mobility spectrometry (IMS), which separates charged particles according to their size-to-charge ratio and their interactions with the gas phase, was used as a standalone technique. In differential ion mobility spectrometry (DMS), ions experience a different mobility between the low and high field portion of the oscillating field applied between parallel planar electrodes and a specific compensation voltage $(\mathrm{CoV})$ is required to avoid their neutralization on the electrodes. Therefore each analyte that passes through the cell needs a specific compensation voltage to go through. Up to now DMS was mostly used as a noise filter in LC-MS analysis. Recently, it was shown that the addition of gas-phase modifiers (e.g. ethanol, isopropanol) could significantly improve the separation power of DMS. In Fig. 4, the ion mobility separation of eight analytes with the same nominal $\mathrm{m} / \mathrm{z}$ of 316 is illustrated with and without dopants. In this experiment, the $\mathrm{CoV}$ was ramped from -55 to $15 \mathrm{~V}$ and isopropanol showed to be the best dopant for the separation of the eight analytes. With a fixed $\mathrm{CoV}$, the DMS device can be used as a filter to improve assay selectivity but when the $\mathrm{CoV}$ potential is ramping, it can be used as a separation device. This feature was applied to demonstrate real-time 2D separation by LCxDMS hyphenated to mass spectrometry. Further optimization and development work is on-going to enable the application of this technique to the analysis of complex samples. ${ }^{[10]}$

\subsection{Quantitative Selected Reaction Monitoring MS Protein Analysis}

Liquid chromatography coupled to tandem mass spectrometry (LC-MS/MS) has become a very promising analytical tool for the absolute quantitation of therapeutic or endogenous peptides and proteins in biological matrices such as plasma. Most approaches for the absolute quantitative analysis of proteins include the following steps: i) sample preparation, ii) digestion 


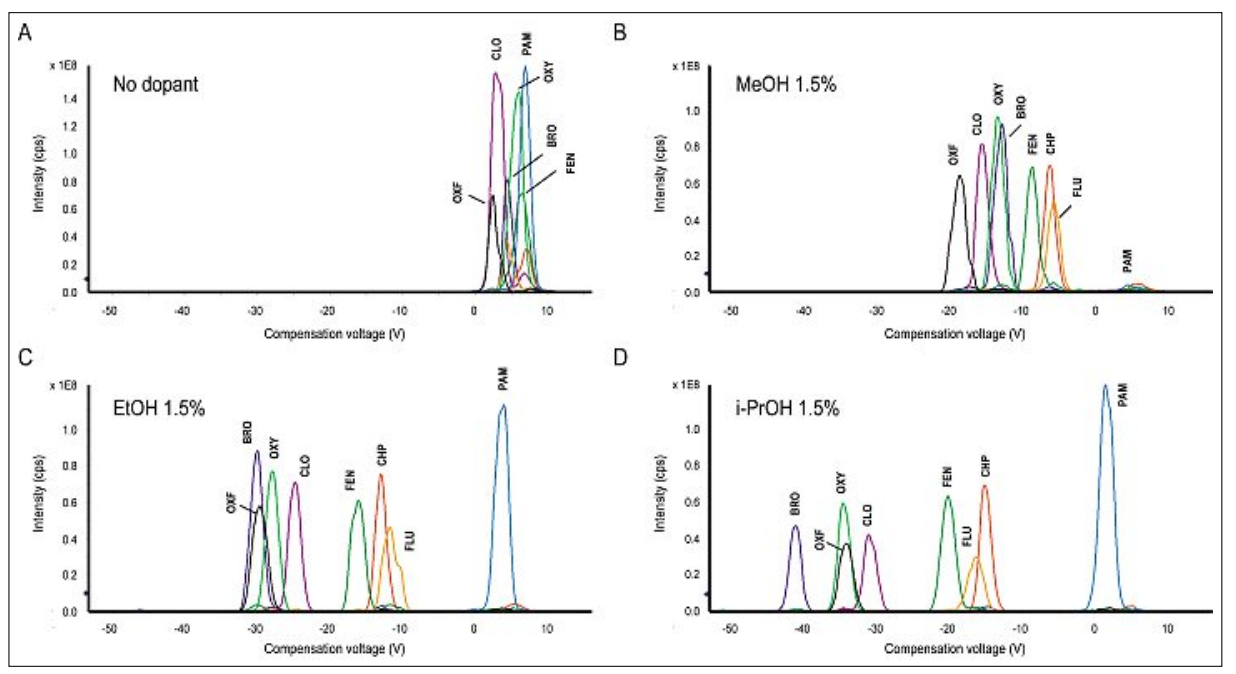

Fig. 4. Separation of the ' 316 mix' by Differential ion Mobility Spectrometry (DMS) coupled to a $\mathrm{QqQ}_{\mathrm{UT}} \mathrm{MS}$ operated in Enhanced Product lon (EPI) scan mode. DMS separation was performed: A) without polar modifier (nitrogen), B) with $1.5 \%$ of $\mathrm{MeOH}$ as modifier, C) with $1.5 \%$ of EtOH as modifier, and $\mathrm{D}$ ) with $1.5 \%$ of $\mathrm{i}-\mathrm{PrOH}$ as modifier. From ref. [10] with permission. Copyright Springer 2011.

of the protein, iii) quantitation of signature peptides as surrogates of the protein, on a triple quadrupole using the selected reaction monitoring mode (LC-SRM/ MS). Ideally, one would like to quantify a large number of proteins with good precision and accuracy as sensitive as possible, with a large dynamic range and in a highthroughput fashion. Analytically, the procedure seems to be quite straightforward but due to the complexity of plasma and the large dynamic range of proteins, the performance of the assay can be jeopardized by many factors. Sample preparation is one of those key factors because on one hand removal of abundant endogenous proteins may result in loss of the protein of interest and on the other hand whole digestion of plasma requires large amounts of trypsin and generates interfering peptides. When large set of samples has to be analyzed, the digestion process needs to be carefully considered. Proteins digestion can also be significantly accelerated with microwaves or at higher temperatures. As for the analysis of small molecules, the use of an adequate internal standard, which follows most of the sample preparation steps and compensate for matrix effects during ionization, is highly desirable and several approaches can be considered. The major outcome of the investigation of accelerating the protein tryptic digestion is that the peptides from the human $\mathrm{mAb}$ were generated at different rates and were classified into three categories: i) the fast forming peptides, ii) the slow forming peptides and iii) the peptides degrading over time. ${ }^{[11]}$ For many monitored peptides, a temperature of $37^{\circ} \mathrm{C}$ with a $750 \mathrm{rpm}$ mixing applied for at least $30 \mathrm{~min}$. provided equivalent results to microwave-assisted digestion, and generally allowed the achievement of an equivalent peptide concentration as an overnight digestion carried out at $37{ }^{\circ} \mathrm{C}$. A serial analytical process electrospray LC-SRM/ MS analysis has a limited sample throughput, whereas in LC-MALDI-MS, the LC separation step can be decoupled from the MS detection and different improvements in sample throughput can be considered. For that purpose, a high-repetition MALDI triple quadrupole linear ion trap is considered as an alternative to LC-SRM/MS for the quantitation of proteins. ${ }^{[12]}$

\section{Acknowledgements}

GH and EV would like to thank present and past members of the Life Sciences Mass Spectrometry group (www.unige.ch/sciences/ pharm/lsmv/index.html) for their significant contributions. They also would like to acknowledge AB Sciex, Dionex, Spark Holland and Shimadzu for their support, as well as Swiss Systemsx.ch initiative, grant IPP-200NIONN and SNF (Sinergia) for funding.

Received: February 20, 2012

[1] L. A. McDonnell, R. M. Heeren, Mass Spectrom. Rev. 2007, 26, 606.

[2] B. Balluff, C. Schone, H. Hofler, A. Walch, Histochem. Cell. Biol. 2011, 136, 227.

[3] P. Kovarik, C. Grivet, E. Bourgogne, G. Hopfgartner, Rapid Commun. Mass Spectrom. 2007, 21, 911.

[4] M. Wagner, E. Varesio, G. Hopfgartner, J. Chromatogr. B 2008, 872, 68 .

[5] G. Hopfgartner, E. Varesio, M. Stoeckli, Rapid Comm. Mass Spectrom. 2009, 23, 733.

[6] T. Porta, C. Grivet, T. Kraemer, E. Varesio, G. Hopfgartner, Anal. Chem. 2011, 83, 4266.

[7] T. Porta, T. Kraemer, E. Varesio, G. Hopfgartner, in 'Surface sampling and differential ion mobility spectrometry for the direct analysis of illicit drug metabolite isomers from tissue sections', 59th Annual Conference on Mass Spectrometry and Allied Topics, Denver, CO, June 5-9, 2011, Denver, USA.

[8] R. Mohamed, E. Varesio, G. Ivosev, L. Burton, R. Ronner, G. Hopfgartner, Anal. Chem. 2009, 81,7677 .

[9] G. Hopfgartner, D. Tonoli, E. Varesio, Anal. Bioanal. Chem. 2011, DOI 10.1007/s00216011-5641-8.

[10] E. Varesio, J. C. Le Blanc, G. Hopfgartner, Anal. Bioanal. Chem. 2011, DOI 10.1007/ s00216-011-5444-y.

[11] A. Lesur, E. Varesio, G. Hopfgartner, $J$. Chromatogr. A 2010, 1217, 57.

[12] A. Lesur, E. Varesio, G. Hopfgartner, Anal. Chem. 2010, 82, 5227. 